\title{
Real Time Implementation of Nodes Self Scheduling Approach for Maximizing Wireless Sensor Network Lifetime
}

\author{
E. Abarna Ignatius ${ }^{1}$, Mrs.F.Vincy Lloyd ${ }^{2}$ \\ ${ }^{I}$ (ECE, D.M.I College of Engineering / Anna University, India) \\ ${ }^{2}$ (Associate Prof-ECE, D.M.I College of Engineering/ Anna University, India)
}

\begin{abstract}
To face the short lifetime of the WSN, the objective is to optimize energy consumption while maintaining the full sensing coverage. A major technique to save the energy is to use a wake-up scheduling protocol through which some nodes stay active whereas the others enter sleep state so as to conserve their energy. This technique presents an original algorithm for node self-scheduling to decide which ones have to switch to the sleep state. The novelty is to take into account the best shortest path among the nodes in wireless sensor network .Hence, the sensed data from the network is transmitted over the best shortest path by verifying the updates in the routing table. To verify and evaluate the proposed algorithm, simulations have been conducted and have shown that it can contribute to extend the network lifetime. A comparison with existing works is also presented and the performance gains are highlighted.
\end{abstract}

Keywords: wireless sensor networks, simultaneous wake up scheduling, best shortest path.

\section{INTRODUCTION}

Wireless sensor networks (WSNs) consist of tiny devices, which have a battery, a sensor, a microprocessor and a radio transmitter component. The application area of WSNs can be classified into two general classes: monitoring applications and tracking applications. While the rest class of applications includes habitat monitoring, building monitoring, machinery monitoring and greenhouse monitoring etc., the second class includes animal tracking, vehicle tracking and goods tracking in supply chains etc. Due to this large range of application area, performance metrics in sensor network are strictly application specific. However, 'unattended operation of the network for long time' or 'long network lifetime' can be determined as a common performance requirement for the most of the applications. In general, network lifetime can be defined as time span until the network is considered non functional. In fact, as in performance metrics, perception of no functionality is also application-specific for sensor networks.

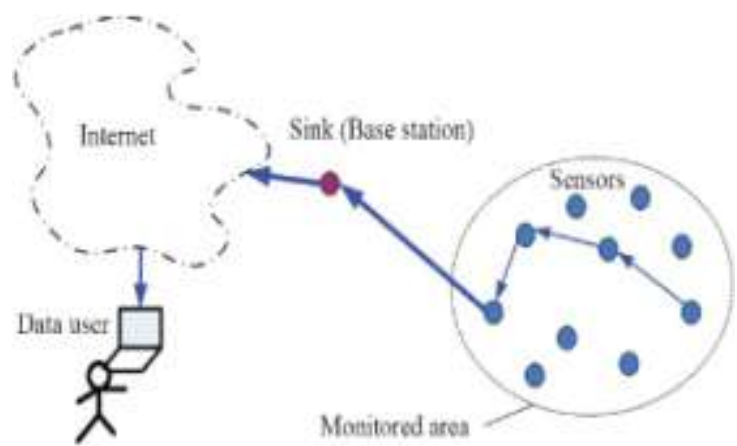

Fig 1: A Basic Wireless Sensor Network

A sensor network can be considered as non-functional if data delivery delay exceeds a threshold, or if the coverage of the monitoring area is less than the desired level, or if the network is partitioned due to the energy deficiency of some bottleneck nodes. Energy depletion of nodes can interrupt communication and, in a worse case, it could cause network partitioning which lead the interruption of monitoring. Unlike ad-hoc networks, recharging or replacing the sensors battery may be inconvenient, or even impossible in some monitoring environments. Therefore the key challenge in the design of wireless sensor network protocols is how to maximize the network lifetime, which is limited by battery energy in sensor nodes, while providing the application requirement. The data aggregation regime in the network (raw data transmission or processed data transmission, periodic or event triggered data gathering [4]); the channel access (MAC) schemes [1]; the routing protocols [3], [2]; and the energy model for transmission (based on the channel model). This paper focuses on maximizing the lifetime of a sensor network from the routing protocols point of view. 
In wireless sensor networks, all nodes share common sensing tasks. This implies that not all sensors are required to perform the sensing task during the whole system lifetime. Turning off some Nodes does not affect the overall system function as long as there are enough working nodes to assure it. Therefore, if we can schedule sensors to work alternatively, the system lifetime can be prolonged correspondingly; i.e. the system lifetime is prolonged by exploiting redundancy. In this work, we present a novel node scheduling scheme, which is used to configure node work status and schedule the sensor on-duty time in large sensor networks. Our design has been driven by the following requirements: first, because it is inconvenient or impossible to manually configure sensors after they have been deployed in hostile or remote working environments, self-configuration is mandated. Second, the design has to be fully distributed and localized, because a centralized algorithm needs global synchronization overhead and is not scalable to large-populated networks. Third, the algorithm should allow as many nodes as possible to be turned off in most of the time. At the same time, it should preserve the initial sensing coverage with minimum "sensing hole", or "blind points". It is ideal if the working nodes can cover the same monitored area as the original one. Fourth, the scheduling scheme should be able to maintain the system reliability, i.e., certain redundancy is still needed. In the proposed approach, each node in the network autonomously and periodically makes decisions on whether to turn on or turn off itself only using local neighbour information. To preserve sensing coverage, a node decides to turn it off when it discovers that its neighbours (sponsors) can help it to monitor its whole working area. To avoid blind point, which may appear when two neighbouring nodes expect each other's sponsoring, a back off-based scheme is introduced to let each node delay its decision with a random period of time.

In this work, we implement the proposed scheme to transmit the data over the sensor network by using the best shortest path by referring the routing table. During the transmission through the shortest path all the nodes are made to be in idle state except the path which transmits the data is placed in active state.

\subsection{Introduction}

\section{COVERAGE PROBLEM}

Before introducing the proposed algorithm, the coverage problem and some related works are presented. The coverage idea was introduced in [2] as a QoS. It is introduced using the fundamental question: 'How well do the nodes observe the physical space?'. Owing to the large variety of the nodes and their applications, the coverage problem is subject to a wide range of interpretations [3]. The coverage problem may be divided into three categories depending on what exactly that you are attempting to monitor. 'Area coverage' [4-6]: the overall goal is to have each location of the interest area within the sensing range of at least one node. 'Target coverage' $[7,8]$ : observes a fixed number of targets. 'Barrier coverage' $[9,10]$ : refers to the detection of movement across a barrier of sensors. This paper considers the area coverage problem. Thus, some related works are detailed in the remaining of this section to better understand the development of the proposed algorithm.

Since the sleeping state is the least energy consuming state, keeping nodes in the sleeping state is a good way to save energy. However, the full area coverage must be ensured when some nodes are sleeping.

Some work as those in $[11,12]$ propose to divide the nodes into disjoint sets, such that every set can individually and successively perform the area monitoring task. These sets are successively activated, and all nodes, not belonging to the active set, will be in the sleep state. Generally, such algorithms are centralized based on a full knowledge of the network topology, which increases the cost of the algorithm. Such solution is not reliable because of the failure of nodes. Indeed, when a node fails, the coverage is no more guaranteed despite the fact that the other nodes belonging to the same set remain working.

As centralized solutions are not adequate to WSNs, specially those including large numbers of nodes, because of their expensive energy cost, distributed and particularly localized algorithms were more privileged.

The algorithm presented in [13] is localized and divided into rounds. Each round begins with a selfscheduling phase followed by a sensing phase. In the self-scheduling phase, nodes investigate the off-duty eligibility rule. Based on the eligibility rule, each node verifies if its sensing area (the set of points that it reaches for sensing) is covered by the union of the sensing areas of its neighbors. Eligible nodes turn off their communication and sensing units, whereas other nodes will perform the monitoring tasks in the sensing phase. To avoid the occurrence of blind points (non-covered points), which can occur when the eligibility rule is simultaneously investigated by neighboring nodes, a back off method is used. Thus, a node that has a longer backoff time will not consider those that have already decided to be inactive (from which it has received a status advertisement message).

A localized probing-based self-scheduling algorithm is proposed in [14]. A node goes back to the sleep state if it receives at least one reply to its probation message (PRB) message otherwise it remains active. The PRB message is broadcast within a probing range $r$ depending on the application's specificity. The problem is that such an algorithm does not guarantee the coverage of the target area. 


\subsection{Connectivity Problem}

In addition to the energy preservation, connectivity is also one of the important issue related to the coverage problem. An important frequently addressed objective is to determine a minimal number of working nodes required to maintain the initial coverage and as this set is connected. An important result, proved by Zhang and Hou [6], states that if the communication range is at least twice the sensing range, a complete coverage of the area implies the connectivity of the working nodes. The authors also observed that an area is completely covered if there are at least two disks that intersect and all crossings are covered. Disk refers to a sensing area of a node and a crossing is an intersection point of the circle boundaries of two disks. Based on these results [6], the authors proposed a localized algorithm for node scheduling. A probabilistic coverage with various sensing models has also been studied in [16-18].

The development of the proposed algorithm is justified by the desire to circumvent the different challenges previously presented, as there are no algorithms, according to our knowledge, which considers all these elements when scheduling the node's activities while preserving the area coverage. Particularly, the energy constraint, which has a major impact on the WSN lifetime, must be taken into account at organizational and decisional levels. On the one hand, the best path should be made active while making all other nodes into idle state . On the other hand, decisions must be made based on local information and the exchange of many messages must be avoided, thus, reducing the overheads and as a consequence, the energy cost of the algorithm.

\section{PROPOSED DESIGN}

\subsection{Problem Statement}

In an sleep-scheduling wireless sensor network, sensors are competing only with manual sleep nodes with the usage of limited active nodes leading to coverage and connectivity problems. In most sensor networks, multiple sensors are deployed to monitor the desired area. In addition to make nodes sleep manually it is efficient to make a simultaneous wakeup scheduling approach such that only the nodes transmitting the data is made to be active were the rest of the nodes are in idle state.For transmission of next data the next best path is chosen through the routing table.

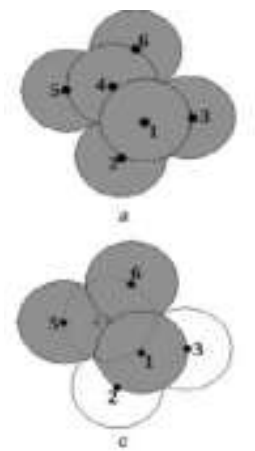

a Original sensing area covered by nodes $1-6$

b Node 1 turns off itself by the eligibility rules

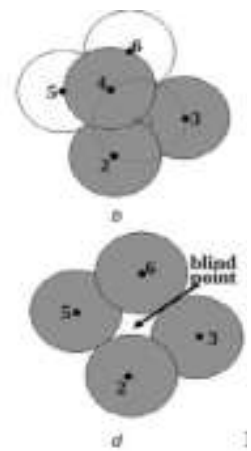

c Node 4 turns off itself by the eligibility rules

d Occurence of a blind point

Fig 2: Blindpoints

If multiple nodes pick the same path to transmit at the same time and it leads to network congestion, collision at receiver end; which ends up in lots of energy wastage. There is possible for the blind points to occur.

\subsection{Problem Formulation}

The wireless sensor network is modelled as a Nodes, and $\mathrm{L}$ is the set of the links between them. If two nodes $\mathrm{n}$ and $\mathrm{m}$ are connected by a directed link, they can communicate with each other. Direction of the link is determined from farther node to the closer one to the sink node. For the connection of node $\mathrm{n}$ with any node $\mathrm{m}$, a variable is defined to represent the total flow on an outgoing links of $\mathrm{n}$ as $f l_{\mathrm{mm}}$ and this variable also represents the total flow on an incoming link of $m$.

For the sake of notation, let variables $\mathrm{f} 1$ and $\mathrm{f} 2$ represent the total amount of flow (fl) during the network lifetime on the outgoing (On) and incoming (In) links of a node n, respectively, and depicted as follows:

$\mathrm{f} 1=\sum_{\text {Inmeon }}$ flnm $\mathrm{n}, \mathrm{m} \in \mathrm{N}, \mathrm{L} \in \mathrm{L} \square$

$\mathrm{f} 2==\sum_{\text {lnm }} \in \ln$ flmn $\mathrm{n}, \mathrm{m} \in N_{s}, M \in L$ 
The lifetime, $T_{n}$, of the individual node $\mathrm{n}$ is formulated as being the sum of the time spent in transmission, reception, and idle mode of operation, and it is depicted as follow:

$$
T_{n}=\mathrm{f} 1 . t_{t x x}+\mathrm{f} 2 \cdot t_{v x}+t_{i d l e}^{n} \mathrm{n}, \mathrm{m} \in N, L \in L
$$

In the above equation, $t_{t x}$ and $t_{v x}$ are constant and represent the transmission time and the reception time of a data packet respectively, hence f1. $t_{t x}$ Represent the total transmission time and f2. $t_{v x}$ represent the total reception time for a node $\mathrm{n}$ at the end of its lifetime. Note that $t_{\mathrm{tx} x}$ and $t_{v x}$ will differ based on the data packet size and speed of the transceiver. $t_{\text {idle }}^{\mathrm{n}}$ Is a variable and represents the total idle time of the node spent in its whole lifetime.

\section{SYSTEM DESIGN}

Simultaneous wakeup scheduling of nodes in transmission of the data to the destination by using the best suitable path is the main focus in this work. Concurrent usage of the same path by multiple sensor nodes would lead to collision and retransmission of data packets resulting in degradation of the network efficiency and waste of energy in sensor nodes. AODV is a protocol by which a routing table is created which has the updates of all the neighbour nodes along with the distance, energy, bandwidth of the neighbouring nodes . Decisions are made by the routing table to chose the best for data transmission. Another important function handled by the data link layer is flow control. The sender and the receiver come to an understanding on how much of data can be sent, so that it is convenient for both the sender and the receiver to optimize the bandwidth utilization, this function is called flow control. Our proposed system has been classified into three phases based on the functionalities such as initialization, data collection and communication phases.

\section{Initial phase}

1. Initially all nodes are location aware and know their distance from the sink.

2. On initialisation every sensor node broadcasts a control message (ADV) which includes source ID, residual energy and radius .

3. On receiving each node creates a neighbourhood table (routing table) and updates distance from the neighbour node and residual energy.

\section{Data Collection Phase}

1. In this phase when the data is sensed, the nodes verify the routing table and chooses the best path for transmission.

2. Depending upon the data sensed ,based on it the best path is chosen for transmission.

3. Based on the size of the data and the updates in the routing table the first best path is chosen.

\section{Communication phase}

1. The sensed data is transmitted to the destination by referring the routing table to chose the first best routing path and turning the remaining nodes that are away from the path to idle state .

2. Since the path is organised based on the bandwidth of the nodes , based on the size of the packet the best active path is chosen from the routing table.

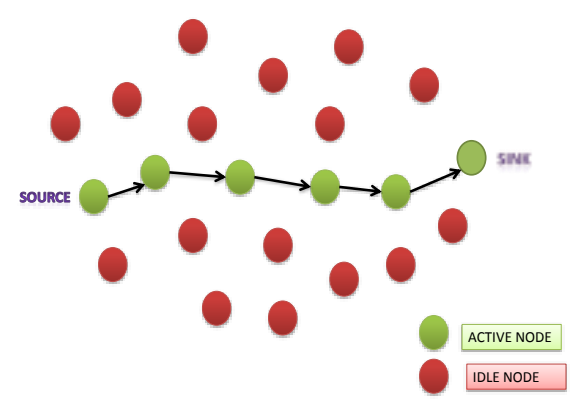

Fig 3: Data Transmission using the best shortest path

In the initialization phase, nodes in the network introduce themselves by broadcasting the ADV message which contains the source Id, location info, residual energy and best path schedule information to immediate neighbours which are within the communication radius. 
Then a routing table is created by the AODV protocol based on the information obtained to chose the shortest routing path to perform the load balancing. The MAC protocol uses the sleep schedule information to control the medium access and reduce the energy wastage. The energy efficient design and implementation of MAC protocol depends on the capabilities and features of the underlying radio transceiver and microcontroller. Our MAC protocol uses the power of in-network data processing and communication tasks simultaneously to determine the total energy required to transmit the amount of sensed data. In-network data processing greatly reduce the energy consumption compared to transmitting all the raw data to the end node[5]. Proposed system integrates the shortest path routing algorithm and stores the routing table and uses the best shortest paths to route the control message and payload transmission. If the first shortest route is not feasible due to active/idle duty cycle then it selects the second best shortest path until the first best route is available.

In case of huge data transfer from multiple sensors, data packets can be converted into small packets and can be sent across using the shared transmission medium. Smaller data packet from multiple source can be placed in any order as long as there is available bandwidth to carry it. Each data packet contains the destination address and sequence number and it will help the receiver node to reconstruct the node data if required and sent to the proper recipient. Our method utilize the full extent of available network bandwidth for data transmission and it helps in reducing the waiting time of the contender nodes.

\section{Simulation RESUlTS}

We have following assumptions in our simulation setup:

1) Sensor nodes are static and there is only one sink node.

2) Nodes are location aware and know their distance from the sink node.

We used the simple energy model explained in [19] for energy consumption evaluation. The energy consumption for sending $l$-bits data to distance $\mathrm{d}$ is ETx and energy consumption for receiving $l$-bits data is ERx The energy model used as shown in the following equations

$\operatorname{ETx}(l$-bits, d $)=l$-bits $(\operatorname{Edpb}+\xi \mathrm{fs} * \mathrm{~d} 2)$

And energy for receiving the $l$-bits data bit is equal to

$\operatorname{ERx}(l$-bits $)=l$-bits $*$ Edpb

Where $\xi \mathrm{fs}$ is constant and $\xi \mathrm{fs}=100 \mathrm{pJ} / \mathrm{bit} / \mathrm{m} 2$ and $\mathrm{Edpb}=50 \mathrm{~nJ} / \mathrm{bit}$, Edpb is energy dissipated per bit .

The parameters involved in the process of deployment of nodes and nodes scheduling process with the details of energy consumption is given by the simulation in NS2.

Channel : Wireless channel

Propogation : Two way propogation

Antenna : Omni antenna

No. of nodes : 25

Routing protocol :AODV

Energy : 100 joules

Packet Size : 5000

Network : Phy/wireless Phy

Queue length: 5

Following simulator shows the deployment of nodes in wireless sensor network using NS2 network simulator.

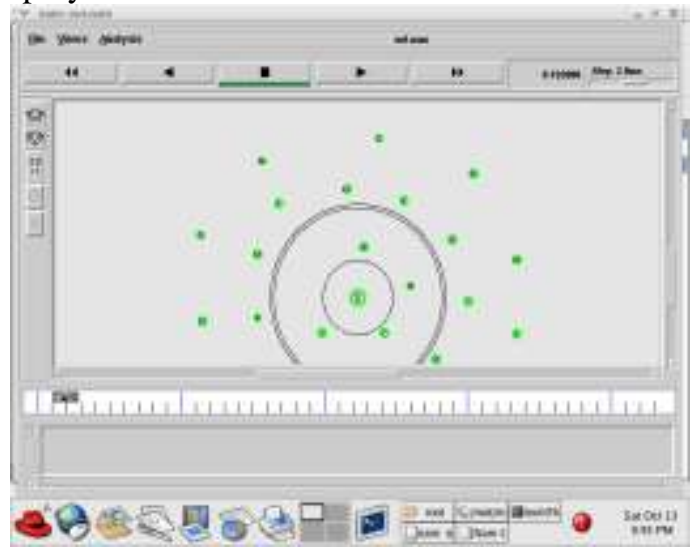

Fig 4: Deployment of nodes in WSN 
Using the NS2 network simulator sleep nodes are used to conserve energy .So by manually turning the nodes to sleep state and performing the data transmission by the active nodes that stay awake.

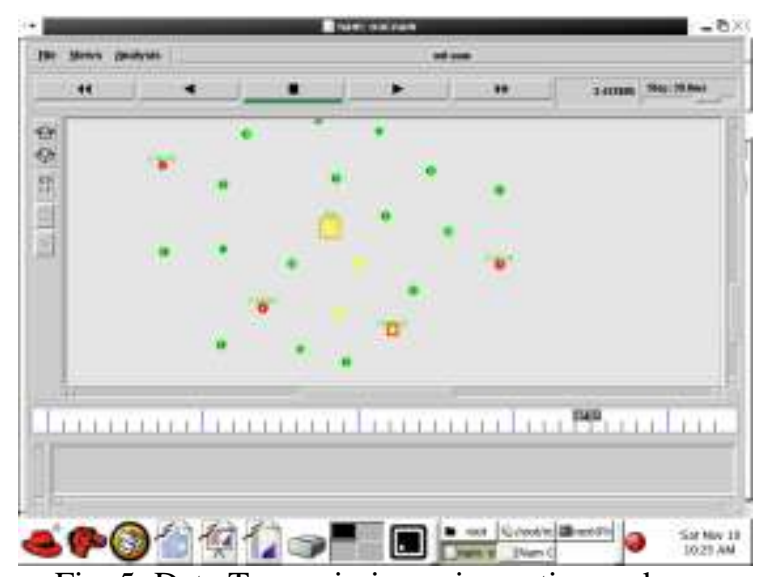

Fig 5: Data Transmission using active nodes

A final simulation using the best shortest path is performed with the representation of the graph specifying the energy, delay and analysis of the system. And also the problems of existing system are addressed.

\section{Conclusion}

In this paper, the problems of energy conservation and full sensing coverage in large WSNs where nodes are randomly deployed have been addressed. An original technique has been introduced based on simultaneous wake-up scheduling concept allowing one to extend the lifetime of the WSN.

Proposed technique is based on using the routing table to choose the best active path for data transmission while engaging all other nodes to idle state. First main feature of this technique is, it is possible to choose an alternate path through the routing table based on the size of the packet. Each best shortest path is formed based on the bandwidth of each node. Second main feature is that a proper understanding is maintained between the source and destination such that the packet is divided and transmitted through the nodes based on the bandwidth of the nodes. Third feature is that if an attacker is present the IDS protocol is used to identify the attacked node and chooses the next best shortest path from the routing table. This has contributed to extend the WSN lifetime.

\section{REFERENCES}

[1] I. Demirkol, C. Ersoy, and F. Alagoz. Mac protocols for wireless sensor networks:a survey. Communications Magazine, IEEE, 44(4):115-121, april 2006.

[2] Jamal N. Al-karaki and Ahmed E. Kamal. Routing techniques in wireless sensor networks: A survey. IEEE Wireless Communications, 11:6-28, 2004.

[3] Jiageng Li, D. Cordes, and Jingyuan Zhang. Power-aware routing protocols in ad hoc wireless networks. IEEE Wireless Communications, 12:69- 81, 2005.

[4] D Niculescu. Communication paradigms for sensor networks. IEEE Communications Magazine, 43(3):116$122,2005$.

[5] Meguerdichian, S., Koushanfar, F., Potkonjak, M.,Srivastava, M.B.: 'Coverage problems in wireless ad-hoc sensor networks'. INFOCOM 2001: 20th Annual Joint Conf. IEEE Computer and Communications Societies, 2001, vol. 3, pp. 1380-1387

[6] Thai, M.T., Wang, F., Hongwei Du, D., Jia, X.:"Coverage problems in wireless sensor networks: designs and analysis', Int. J. Sens. Netw., 2008, 3,(3), pp. 191-200

[7] Ammari, H.M., Giudici, J.: 'On the connected k- coverage problem in heterogeneous sensor nets:the curse of randomness and heterogeneity'.ICDCS '09: Proc. 2009 29th IEEE Int. Conf. on Distributed Computing Systems, 2009, pp. 265-272

[8] Wang, X., Xing, G., Zhang, Y., Lu, C., Pless, R.,Gill, C.: 'Integrated coverage and connectivity configuration in wireless sensor networks', ACM Trans. Sens. Netw., 2005, 1, (1), pp. 28-39

[9] Zhang, H., Hou, J.: 'Maintaining sensing coverage and connectivity in large sensor networks', Ad Hoc Sens. Wirel. Netw., 2005, 1, (2) pp. 89-124

[10] Chang, J.-H., Tassiulas, L.: 'Energy conserving routing in wireless adhoc networks'. INFOCOM 2000: 19th Annual Joint Conf. IEEE Computer and Communications Societies, 2000, pp. 22-31 
[11] Zhang, H., Wang, H., Feng, H.: 'A distributed optimum algorithm for target coverage in wireless sensor networks'. Asia-Pacific Conf. On Information Processing, 2009, pp. 144-147

[12] Chen, A.: 'Designing localized algorithms for barrier coverage'. Proc. ACM MobiCom'07,2007

[13] Osmani, A., Dehghan, M., Pourakbar, H., Emdadi, P.: 'Fuzzy-based movement-assisted sensor deployment method in wireless sensor networks'. Int. Conf. on Computational Intelligence, Communication Systems and Networks, 2009, pp. 90-95

[14] Cardei, M., Thai, M.T., Yingshu, L., Weili, W.:'Energy-efficient target coverage in wireless sensor networks'. INFOCOM 2005: $24^{\text {th }}$ Annual Joint Conf. IEEE Computer and Communications Societies, 2005, vol. 3, pp. 1976-1984

[15] Slijepcevic, S., Potkonjak, M.: 'Power efficient organization of wireless sensor networks'. ICC 2001: IEEE Int. Conf. on Communications, 2001, pp. 472-476

[16] Tian, D., Georganas, N.D.: 'A coverage preserving node scheduling scheme for large wireless sensor networks'. WSNA'02: Proc. First ACM Int. Workshop on Wireless Sensor Networks and Applications, 2002, pp. 32-41

[17] Ye, F., Zhong, G., Lu, S., Zhang, L.: 'Peas: a robust energy conserving protocol for long-lived sensor networks'. Proc. of Int. Conf. on Distributed Computing Systems (ICDCS), 2002, pp. 28-37

[18] Chih-fan, H., Mingyan, L.: 'Network coverage using low duty-cycled sensors: random \& coordinated sleep algorithms'. IPSN'04: Proc.Third Int. Symp. on Information Processing in Sensor Networks,2004. 